\title{
Survival of very low birth weight children and contributing factors: A study from Tehran, Iran
}

\author{
Ramin Mozafari Kermani ${ }^{1 *}$, Jila Sadighi ${ }^{1}$, Abbs Habibollahi ${ }^{2}$, Mahmoud Tavousi ${ }^{1}$, Ali Asghar Haeri Mehrizi ${ }^{1}$, Raheleh Rostami $^{1}$ \\ 1. Health Metrics Research Center, Iranian Institute for Health Sciences Research, (ACECR), Tehran, Iran \\ 2. Ministry of Health and Medical Education- Office of Population and Family, Tehran, Iran
}

Received: 1 May 2021

Accepted for publication: 30 August 2021

[EPub a head of print-20 September 2021]

Payesh: 2021; 20 (5): 589- 598

\begin{abstract}
Objectives: Birth of an infant with very low birth weight (VLBW) (weight less than 1500 grams) is a major cause of deaths and complications in infancy and childhood. Despite recent progressions in medical care and treatment for VLBW children, the mortality rate is high in this group of children. The present study was performed to better identify the survival rate of these children and the problems that affect the survival of this group of infants in the short and long term.

Methods: The study population was all infants weighing less than 1500 grams or infants who borne at less than 32 weeks of pregnancy. Infants whom birth occurred from the beginning to the end of 2018 in hospitals in Tehran were included. Information about these children was provided by the Family and Population Health Office of the Ministry of Health and Medical Education. Sampling was non-random and all information about the infants under study was recorded in a questionnaire. All data from the study were entered into SPSS-22 software and the results were presented using descriptive and analytical statistics. Survival analysis was performed by Kaplan-Meier test and the effect of variables on survival was evaluated using multivariate Cox model.

Results: A total of 338 children were studied. Based on the results of cox regression models, the probability of one-month survival of children was about $68 \%$ and the probability of one-year survival was about $60 \%$. Of the 10 variables affecting the survival of VLBW infants and children under study, 6 variables of maternal education, number of pregnancies, parent and infant insurance, gestational age, birth weight and maternal vaccination before pregnancy were significantly related with the death of infant $(\mathrm{P} \leq 0.05)$.

Conclusion: The findings suggest one of the effective strategies for improving VLBW infant survival rate is encouraging women to continue their education, vaccinating mother before pregnancy, insuring family members and preventing multiple pregnancies.
\end{abstract}

Key words: Infants, Very low birth weight, Survival rate

\footnotetext{
* Corresponding author: Health Metrics Research Center, Iranian Institute for Health Sciences Research, (ACECR), Tehran, Iran

E-mail: Mozafari_ramin@yahoo.com
} 


\section{ميزان بقاى كودكان بسيار كم وزن بدو تولدو عوامل موثر بر آن در شهر تهران 99 |}

رامين مظفرى كر مانى' ‘. زيلا صديقى'، عباس حبيب الهى '، محمود طاووسى'؛ على اصغر حائرى مهريزى'، راحله رستمى'

1. مركز تحقيقات سنجش سلامت، يزوهشكده علوم بهداشتى جهاددانشَاهى، تهران، ايران

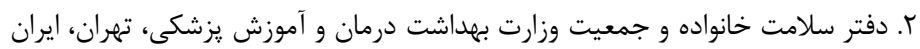

جكيده

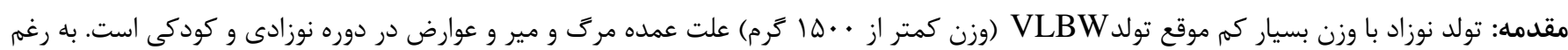

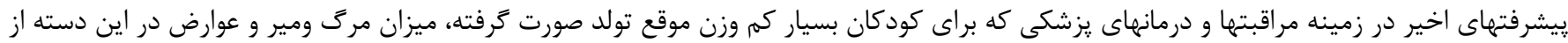

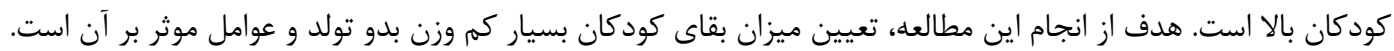

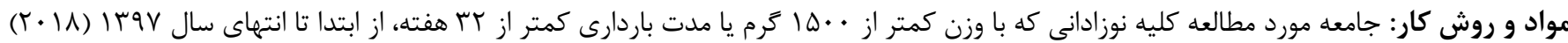

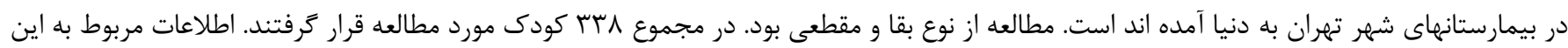

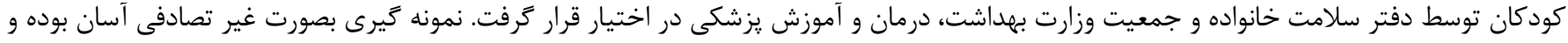

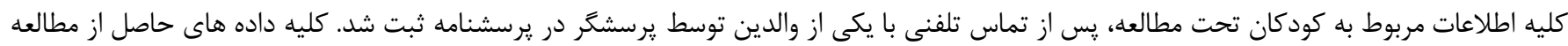

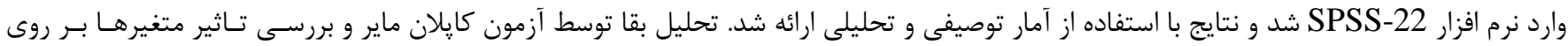

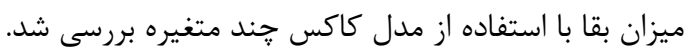

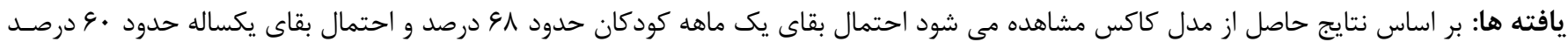

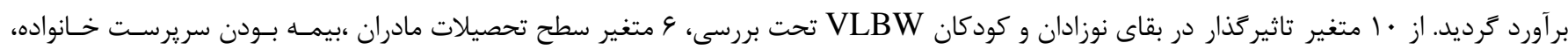

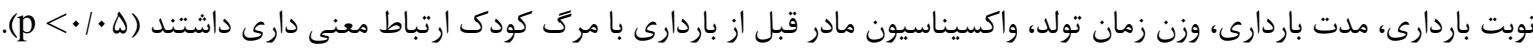

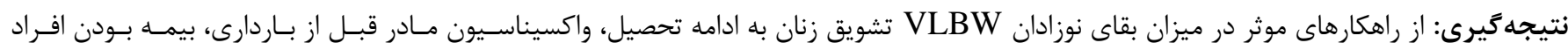

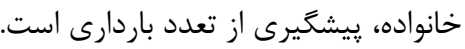

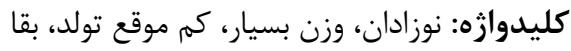




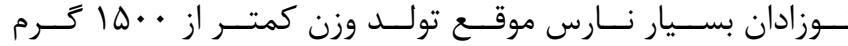
شد. نمونه كيرى به صورت غير تصادفى آسان بوده و كليه اطلاعـات

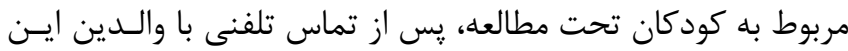

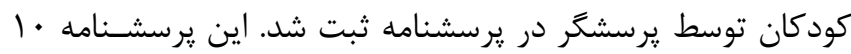

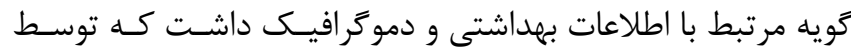

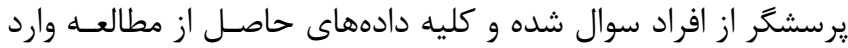

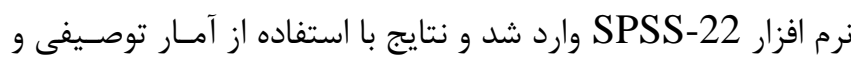

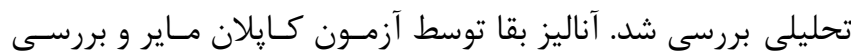

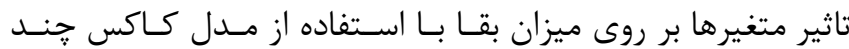

متغيره بررسى شد.

يافتهها

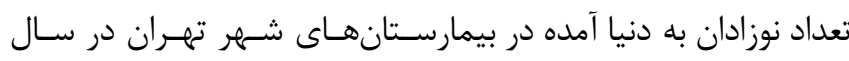

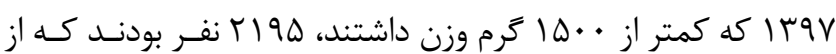

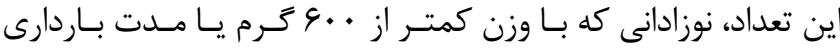

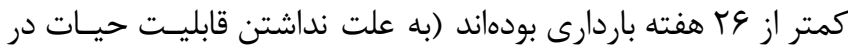

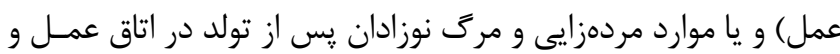

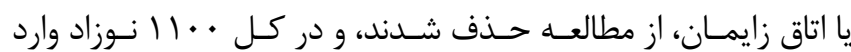
مطالعه شدند كه از اين تعداد مواردى نيز طى تماس بار شماره تمـاس جواب ندادند، و مواردى نيز شماره تلفن آنهـا بـهـ اشـتباه و يـا شـماره

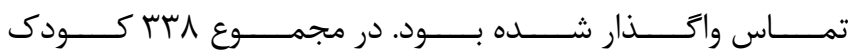

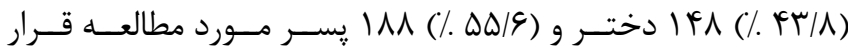
كرفتند. از ^ متغير تاثير كذار در بقاى نـوزادان و كودكـان VLBW

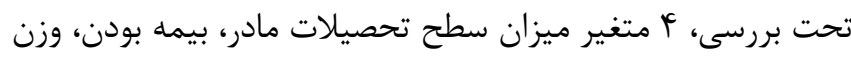

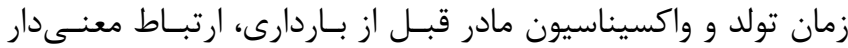

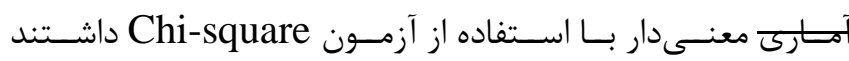

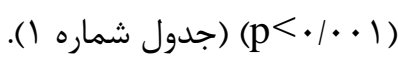

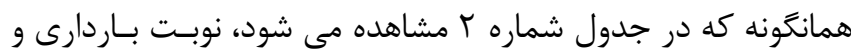

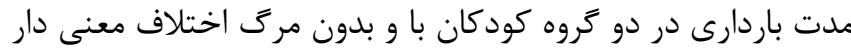

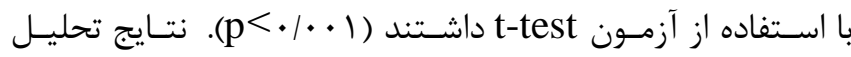
ركرسيون لوجيستيك در جدول شـماره ب نشـان داده شــــ اسـت. نتايج نشان مى دهد متغيرهايى كه در تحليل تكى متغيـره معنسى دار

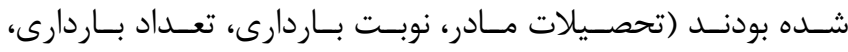
واكسيناسيون مادر قبل از باردارى، وزن زمان تولـد، بيمـهـ خـانوار)، فقط متغيرهاى نوبت باردارى [ (CI: C

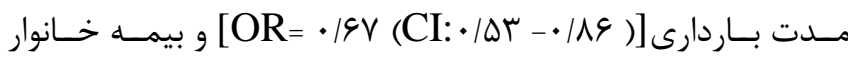

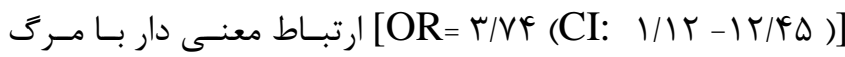

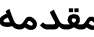

Very low Birst Weight -VLBY موقـع تولـد دارنـد و غالبـاً

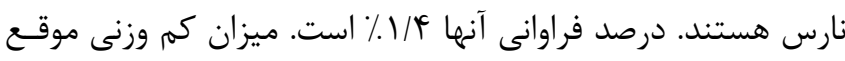

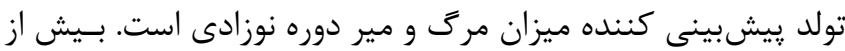

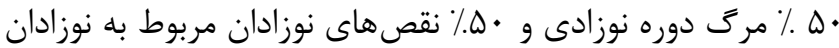

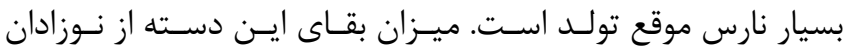

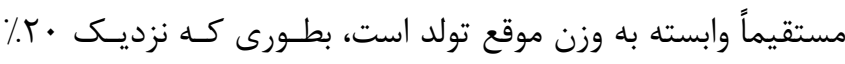

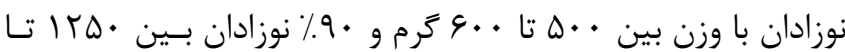

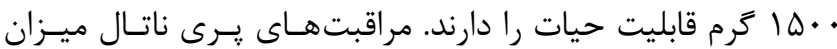
بقاى نوزادان بسيار نارس موقع تولد را بهبود بخشيده در مقايسه بـا بـا

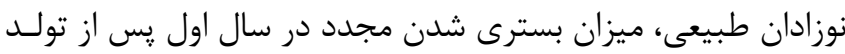

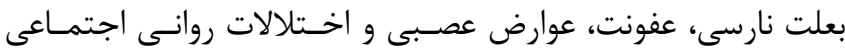

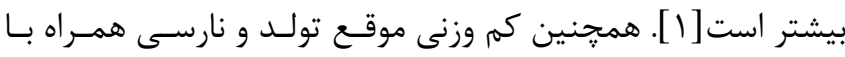

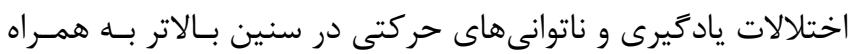

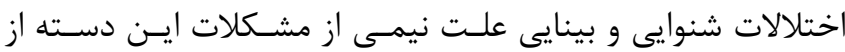

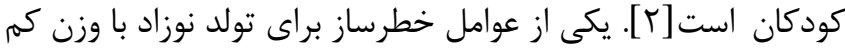

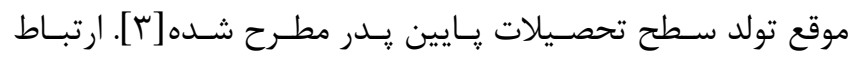
معنى دار بين تولد نوزادان كم وزن بـا سـطح تحصـيلى بـ بـايين مـادر

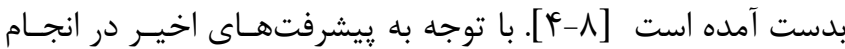

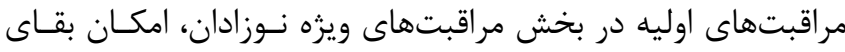

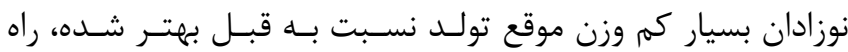
اندازى مراكزى كه بخش مراقبتهاى ويره نوزادان دارد، افزايش تعداد

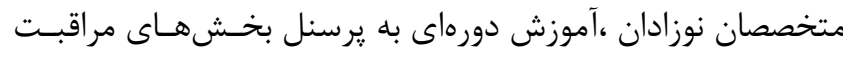

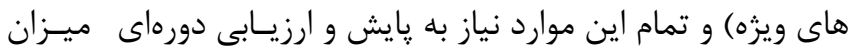

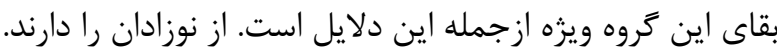

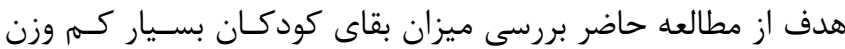

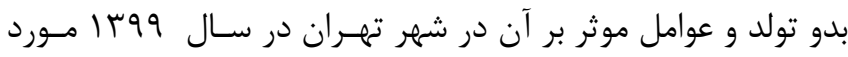
تحقيق قرار كرفت.

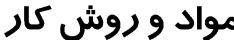

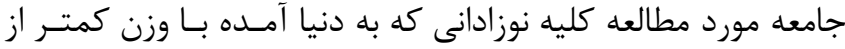

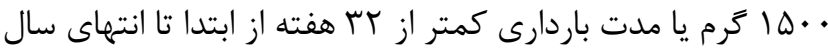

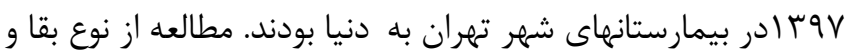

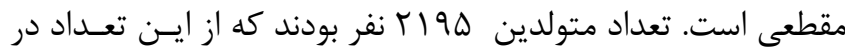

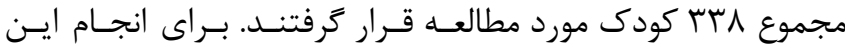

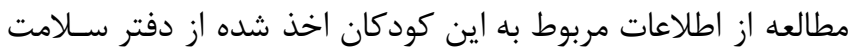

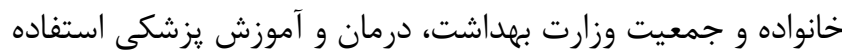


نشريه يزوهشكده علوم بهداشتى جهاددانشكاهى

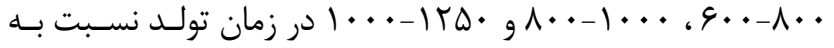

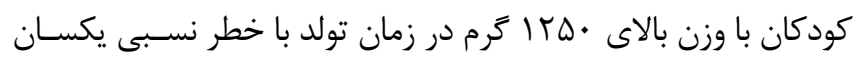

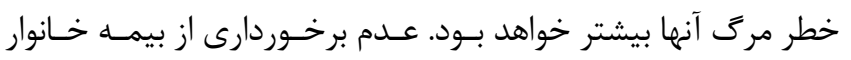

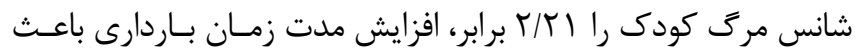

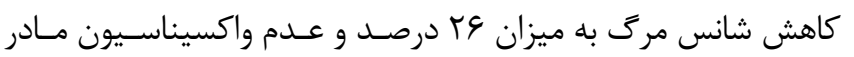

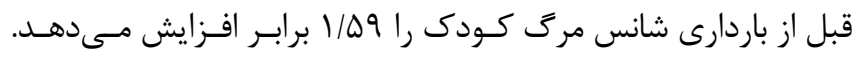
وزن كودى در مخاطره نسبى مرى كودك مـوثر اسـت و ايـن ميـزان براى تمامى وزنهاى دسته بندى شده به يك ميزان خواهد بود.

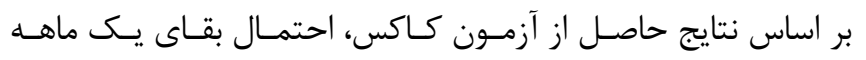

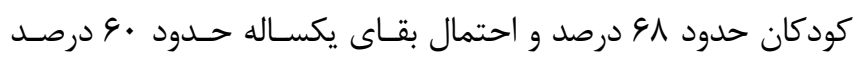

$$
\text { برآورد ₹رديد (نمودار شماره ()). }
$$

كودك داشتند. همانطور كه مشاهده مىشـود، بــا افـزايش هـر يـــ

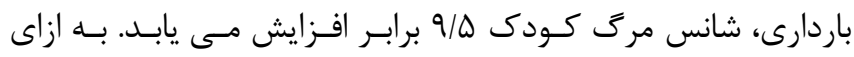

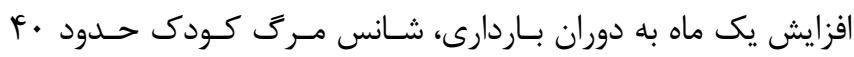
درصد كاهش مى يابد. شانس مـرك در كودكـانى كـه خــانواده آنهـا

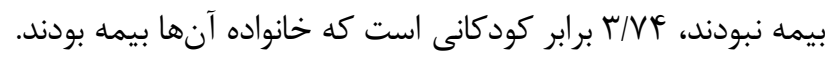

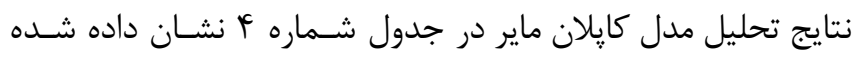

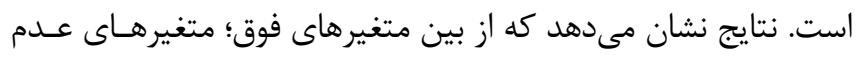

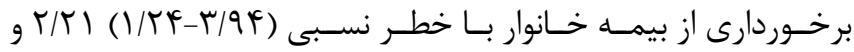

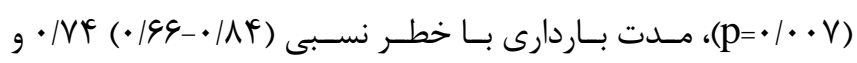

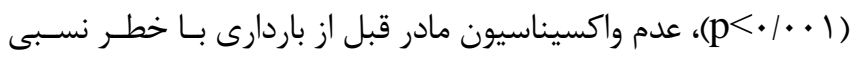

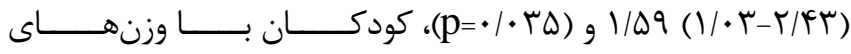
جدول ا: ارتباط تحصيلات يدر، تحصيلات مادر، بيمه سر يرست خانواده، سن مادر هنگًام باردارى، واكسيناسيون مادر، نتيجه زايمان، جنسيت نوزاد و وزن زمان تولد با ميزان مرك كودكان

\begin{tabular}{|c|c|c|c|}
\hline P-value & تعداد (درصد) خير & $\begin{array}{l}\text { تعداد (درصد) } \\
\text { تعلى }\end{array}$ & \\
\hline \multirow{5}{*}{$\cdot / T F$} & & & ميزان تحصيلات يدر \\
\hline & $V(\Gamma / T)$ & $\Lambda(\mathcal{G / V})$ & بيسواد \\
\hline & rq(IV/A) & $T V(T Y / V)$ & زير دييله \\
\hline & $V V(T \Delta / r)$ & MF(TN/G) & دييلم \\
\hline & १९(Kस/A) & $\Delta \cdot(F T / \cdot)$ & دانشَاهى \\
\hline \multirow{5}{*}{$<\cdot 1 \cdot \cdot 1$} & & & ميزان تحصيلات مادر \\
\hline & $\Lambda(r / V)$ & $\mid f(|| / \Lambda)$ & بيسواد \\
\hline & $19(\mathrm{~N} / \mathrm{V})$ & $T Y(1 N / \Delta)$ & زير دييلم \\
\hline & $19(4 \cdot 19)$ & $r q(Y F / F)$ & دييلم \\
\hline & $1 \cdot r(\mathcal{F V} / \cdot)$ & $\Delta F(F \Delta / F)$ & دانشَاهى \\
\hline \multirow{3}{*}{$<\cdot 1 \cdot \cdot 1$} & & & بيمه \\
\hline & $r \cdot r(9 T / \Delta)$ & $91(\Lambda \cdot / \Delta)$ & دارد \\
\hline & $\mid f(\varepsilon / \Delta)$ & $r T(19 / \Delta)$ & 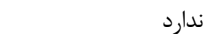 \\
\hline \multirow{5}{*}{$\cdot / r \Delta F$} & & & سن مادر \\
\hline & $r(1 / f)$ & $\cdot(\cdot / \cdot)$ & $<\rangle_{1}$ \\
\hline & $\Delta \backslash(T / T)$ & $r(Y N / \mathcal{F})$ & $r \cdot-4$ \\
\hline & $\mid r \Lambda(q K / \cdot)$ & $G F(\Delta \Delta / r)$ & f. \\
\hline & $T V(\mid T / T)$ & $19(19 / 4)$ & $>r$. \\
\hline \multirow{4}{*}{$.1 . \cdot 9$} & & & واكسيناسيون \\
\hline & $\mid \Lambda \Lambda(\Lambda V / \cdot)$ & $\Lambda \Delta(\vee \Delta / \Gamma)$ & 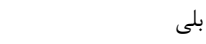 \\
\hline & $r \wedge(\mid K / \cdot)$ & $T \wedge(Y Y / \Lambda)$ & خير \\
\hline & & & نتيجه زايمان \\
\hline \multirow{2}{*}{$\cdot / F \wedge V$} & $119(\Delta F / \Lambda)$ & $99(\Delta 9 / \Delta)$ & يك قلو \\
\hline & $q \wedge(F \Delta / r)$ & $\mathcal{E V}(f \cdot / \Delta)$ & 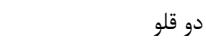 \\
\hline \multirow{2}{*}{$\cdot / \mu \cdot$} & $1 \cdot 1(f \& / T)$ & $F \vee(r q / \Lambda)$ & يسر \\
\hline & $\| V(\Delta r / V)$ & $V I(\varepsilon \cdot / r)$ & دختر \\
\hline & & & وزن (كرم) \\
\hline \multirow{4}{*}{$<\cdot / \cdot \cdot 1$} & $\mid f(g / f)$ & $r \cdot(T V / T)$ & $\wedge \cdots-9 \cdots$ \\
\hline & $\operatorname{cr}(19 / \mathrm{V})$ & rV(Tr/Q) & $1 \cdots-\lambda \cdot 1$ \\
\hline & GI $(\mathrm{TN} / \cdot)$ & TI(19/1) & $\mid r \Delta \cdot-1 \cdot \cdot 1$ \\
\hline & $1 \cdots(f \Delta / q)$ & $T Y(T \cdot / \cdot)$ & $>1 T_{\Delta}$. \\
\hline
\end{tabular}


سال بيستم، شماره هنجم، مهر - آبان ••lf $\int_{4}^{\infty}$

جدول ז: مقايسه نوبت باردارى و مدت باردارى در دو كروه كودكان با و بدون مرى كودكان

\begin{tabular}{|c|c|c|c|}
\hline P-value & ميانكَين (انحراف معيار) & & \\
\hline & & & نوبت باردارى \\
\hline \multirow[t]{4}{*}{.1 .19} & $1 / \Delta V(\cdot / V \wedge r)$ & 119 & بلى \\
\hline & $1 / T \wedge(\cdot \mid q T \xi)$ & 419 & خير \\
\hline & & & مدت باردارى \\
\hline & $r V / \Lambda)(I / \Lambda \mid Y)$ & 111 & بلى بل \\
\hline$<\cdot / \cdot \cdot 1$ & $r q / 9 \cdot(1 / 991)$ & r19 & خير \\
\hline
\end{tabular}

جدول r: ارتباط متغيرها با مرك كودى در مدل ركرسيون لوجيستيك

\begin{tabular}{|c|c|c|}
\hline OR $(95 \% \mathrm{CI})$ & Sig & \\
\hline & & تحصيلات مادر (مرجع: دانشگاهى) \\
\hline$\cdot / V F V(\cdot / r \Lambda \cdot-N / \cdot T V)$ & $\cdot \mid$ evr & 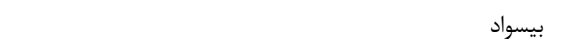 \\
\hline r/r9q(./911-1./191) & $.1 . \Delta F$ & 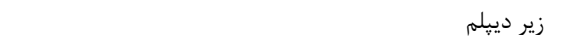 \\
\hline $1 / \cdot r \Lambda(\cdot / F T F-T / \& q \varphi)$ &.$/ 901$ & دييله \\
\hline $9 / \Delta \cdot 1(\tau / \Delta V \Lambda-19 / V r \cdot)$ & $<\cdot / \cdot \cdot 1$ & 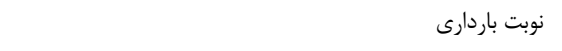 \\
\hline \multirow[t]{2}{*}{$\cdot / 9 V T\left(\cdot / \Delta T_{-}-\cdot / \Lambda \Delta Q\right)$} & $.1 . .1$ & مدت باردارى \\
\hline & & واكسيناسيون مادر قبل از باردارى (مرجع: عدم واكسيناسيون) \\
\hline \multirow[t]{2}{*}{$\cdot / \Delta \& 9(\cdot / r T \Delta-1 / R F 1)$} & $\cdot / r M t$ & 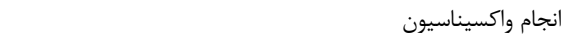 \\
\hline & & وزن زمان تولد (مرجع: •lra> ) \\
\hline 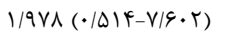 & $\cdot|r r|$ & $4 \cdots-\Lambda \cdot \cdot$ \\
\hline$\cdot \mid \Lambda T \varphi(\cdot / r \Lambda \Delta-T / F \Delta \Lambda)$ & - ves & $\wedge \cdot 1-1 \cdots$ \\
\hline \multirow[t]{2}{*}{ 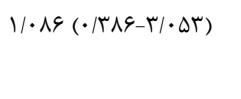 } & $\cdot /$ AVD & $1 \cdots-1$ IN⿴. \\
\hline & & بيمه خانوار (مرجع: داشتن بيمه) \\
\hline H/VTG (I/ITI-IT/AFV) & . & 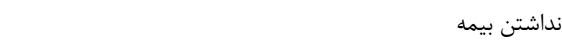 \\
\hline
\end{tabular}

جدول ₹: ارتباط متغيرها با مركى كودى در مدل كايلان - ماير

تحصيلات مادر (مرجع: دانشكاهى)

\begin{tabular}{|c|}
\hline$\cdot 199 \Lambda(\cdot / r T \Delta-1 / F \wedge V)$ \\
\hline $1 / r \cdot \cdot(\cdot \mid q \Lambda F-1 \cdot r / r)$ \\
\hline$\cdot \mid q \cdot V(\cdot / r \Lambda r-\cdot \mid q 9 T)$ \\
\hline $1 / \mid q \psi(\cdot / q \mid f-1 / f \wedge \cdot)$ \\
\hline - $/ V F T(\cdot / 9 \Delta Q-\cdot N F T)$ \\
\hline
\end{tabular}

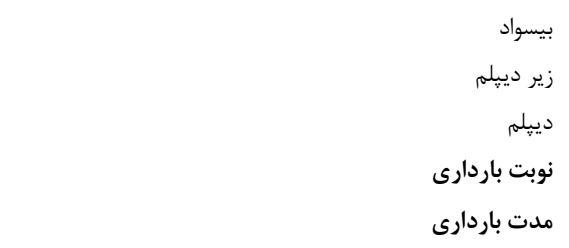

واكسيناسيون مادر قبل از باردارى (مرجع: عدم واكسيناسيون)

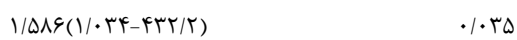

انجام واكسيناسيون

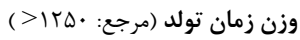

$1 / \mathrm{QV}(\cdot / 99 \Delta-Y \Psi \varepsilon / Y)$

4..-1..

$. / V Y T(\cdot / 910-1 / 9 / 4) \quad \cdot / V Y T$

$\Lambda \cdot 1-1 \cdots$

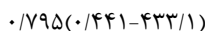

$\cdot$ - FFa

$1 \cdots-1$ I.

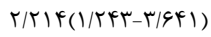

$\cdot / \cdot v$

بيمه خانوار (مرجع: داشتن بيمه) نداشتن بيمه 


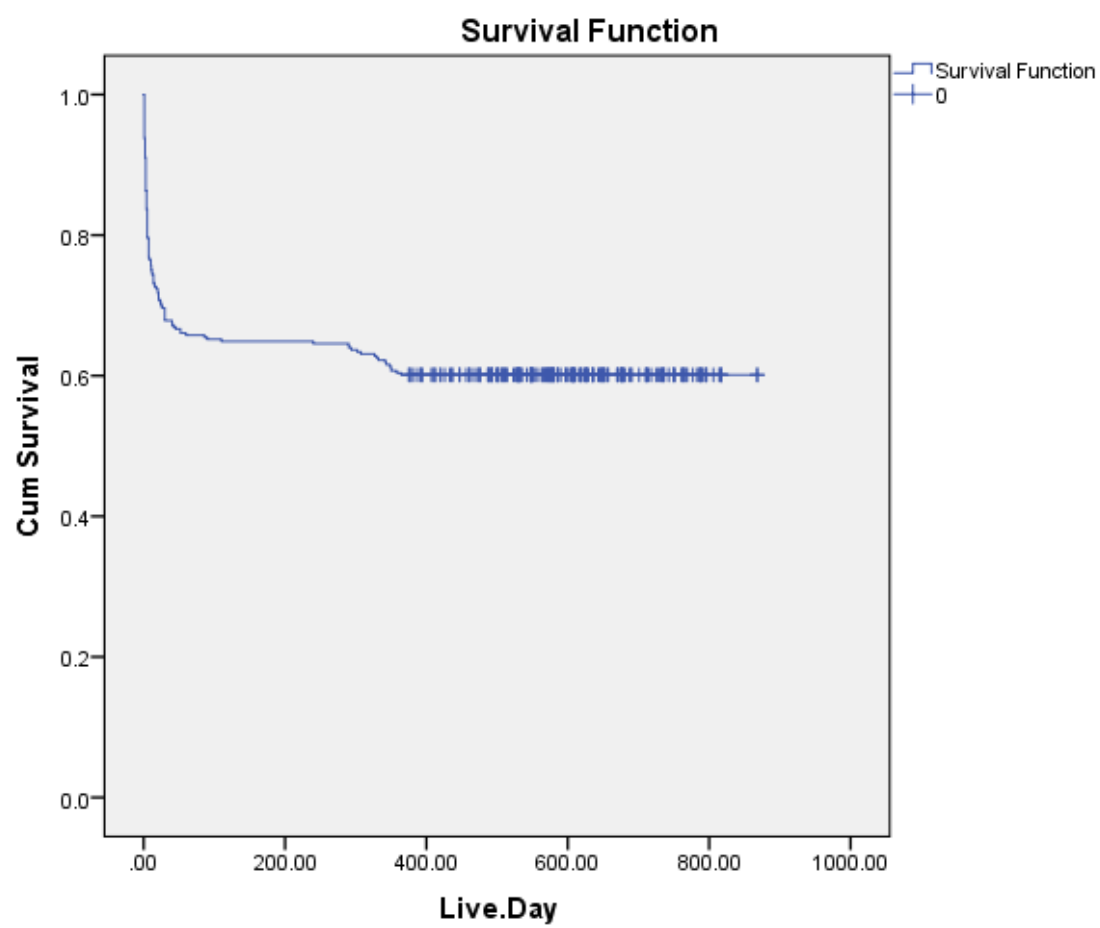

بحث و نتيجه گيرى

در مادران بيسواد // / 1\% بود كـهـ بــا افـزايش سـطح تحصـيلات در

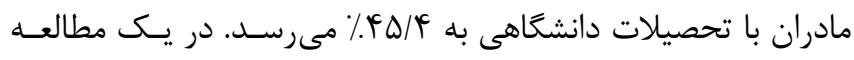

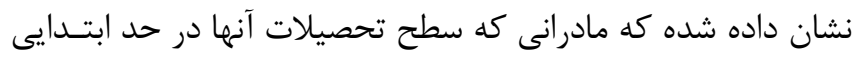
يا كمتر بوده ميزان مرگ و مير نوزادان آنها س \٪ بوده كه در مطالعه

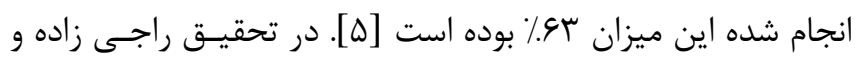

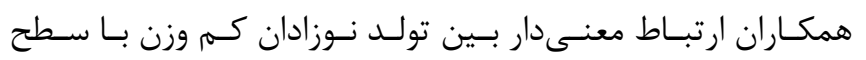

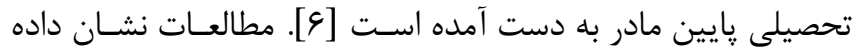

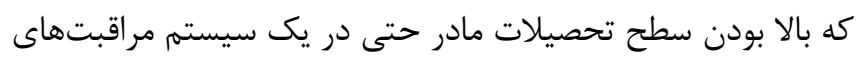

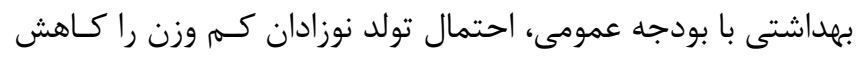

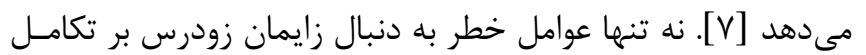

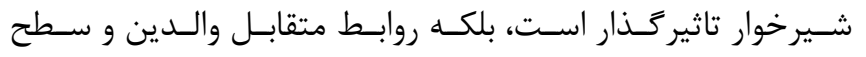

تحصيلات آنها بر تكامل بعدى نوزادان نارس موثر خواهد بود[^].
در اين مطالعه بين سطح تحصيلات پدر و ميزان مرگ و مير نوزادان

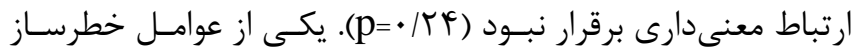

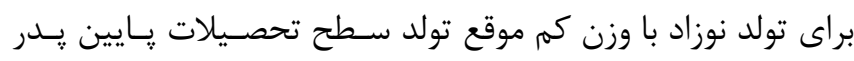

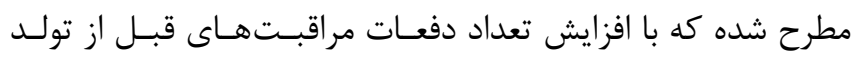

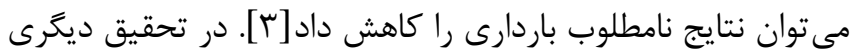

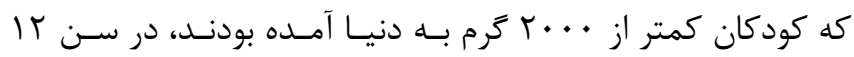

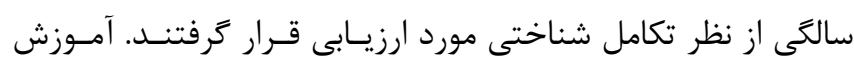

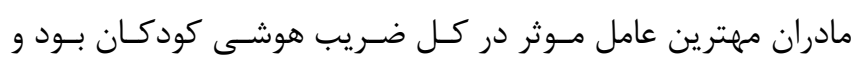

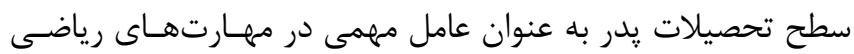
كودكان بوده است [F]. در بررسى انجام شده ارتباط معنى دار بــين

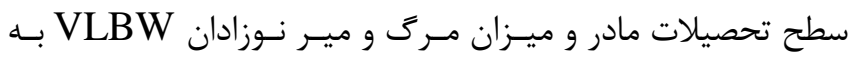

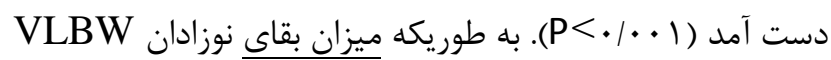


است [ع]]. . در مطالعه انجام شده بين نوبت باردارى و مرك نوزادان ارتبـاط معنسى دارى بـه دسـت آمـــ (PLBW

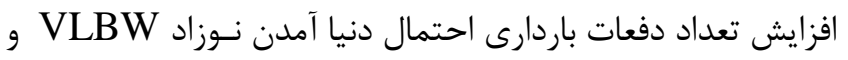

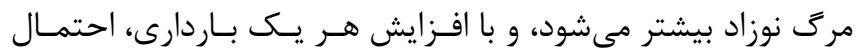

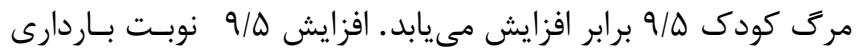

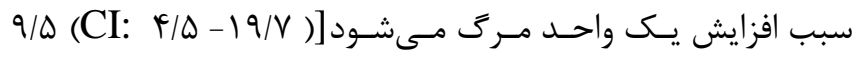
[OR=

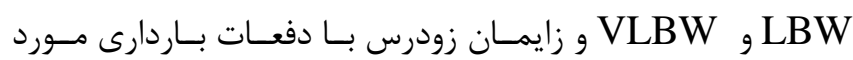

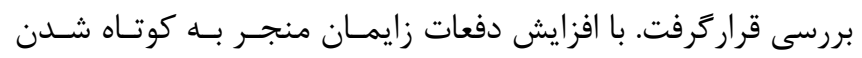

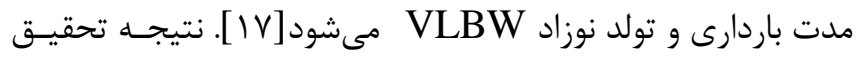

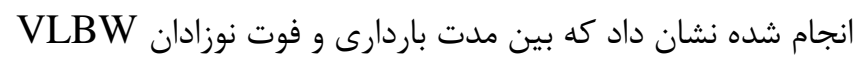

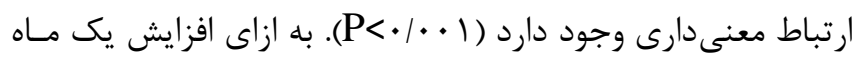

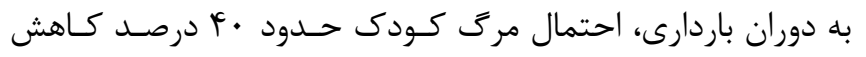

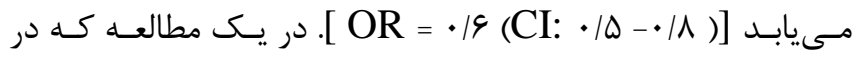

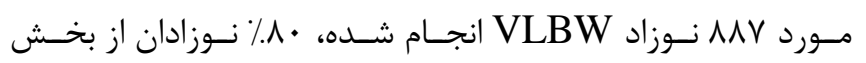

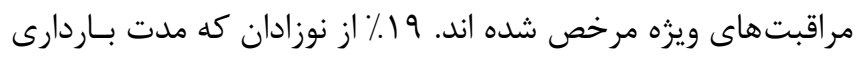

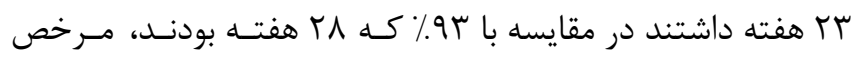
شده اند. ميزان عوارض نوزادان بسترى با افزايش طول مدت بارداردارى

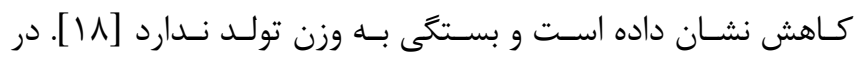

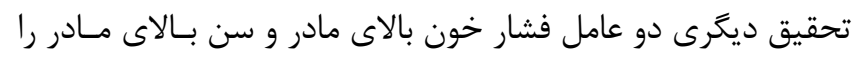

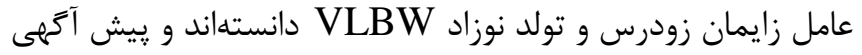

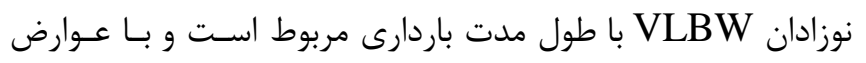

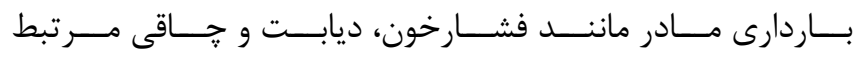

ندانستهاند[19]. از نتايج اين مطالعه وجود ارتباط معنادار بـين واكسيناسـيون مـادر

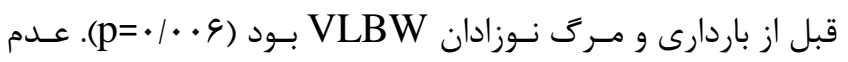

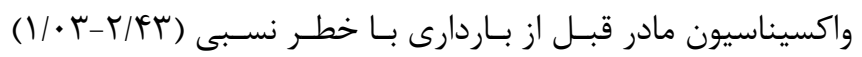

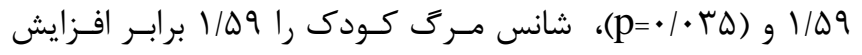

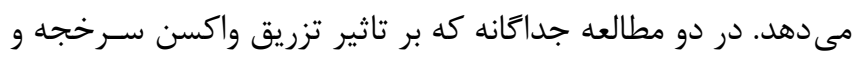

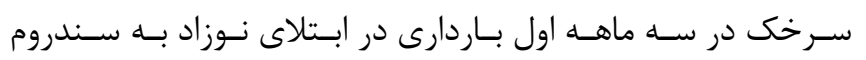

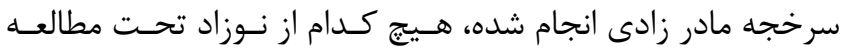

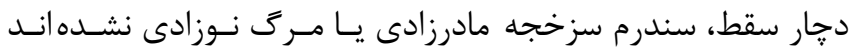

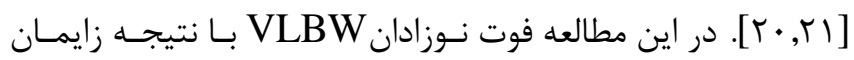

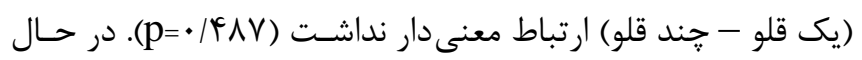

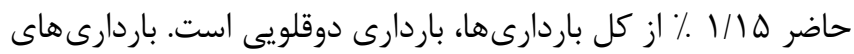

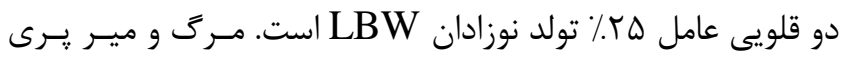

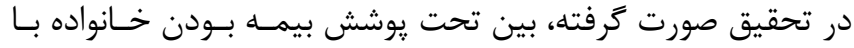

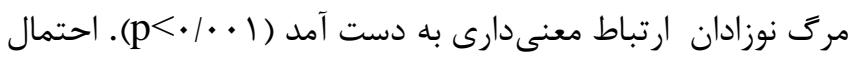

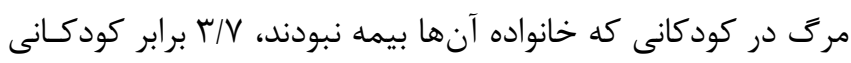

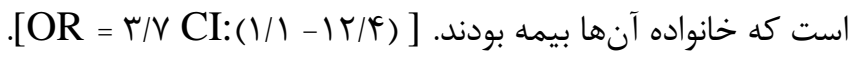

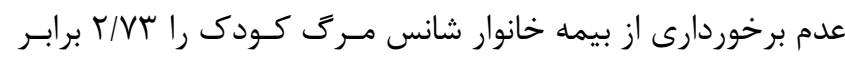

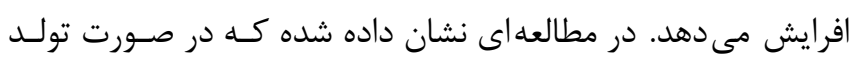

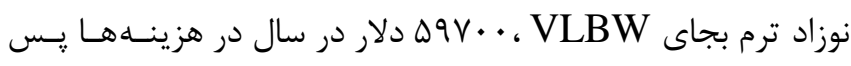

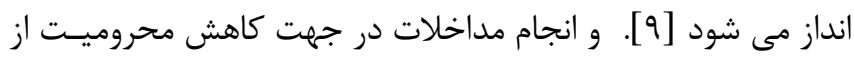

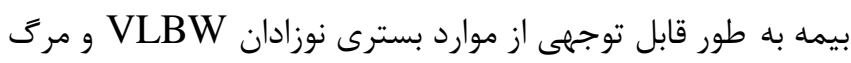

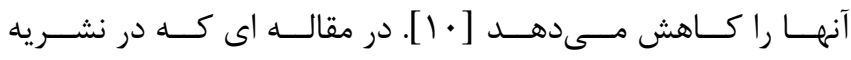
Pediatrics

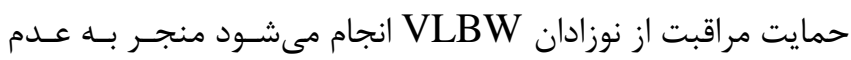

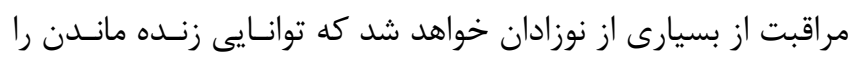

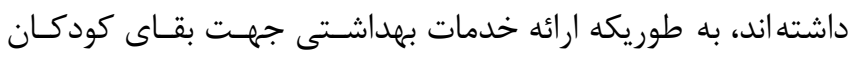

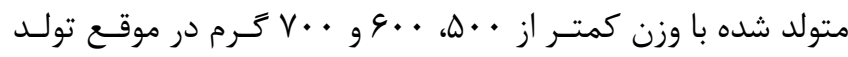

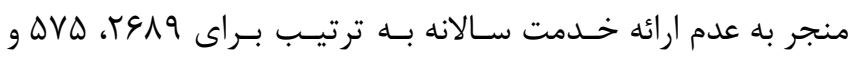

عبا نوزاد ديخر مىشود [11]

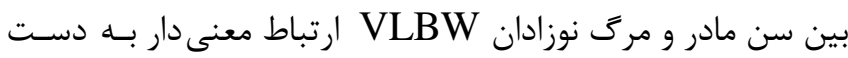

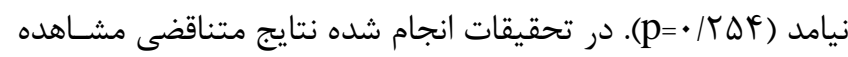

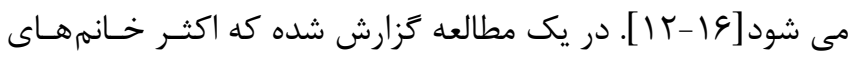

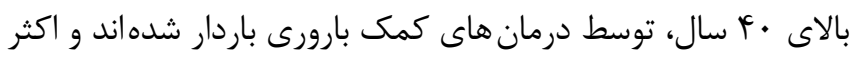

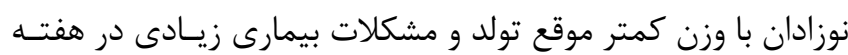

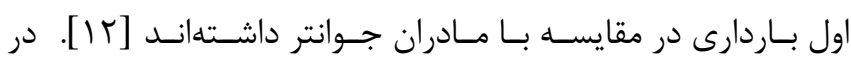

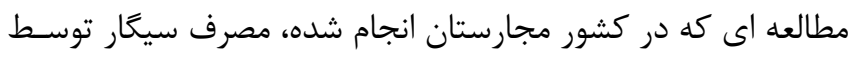

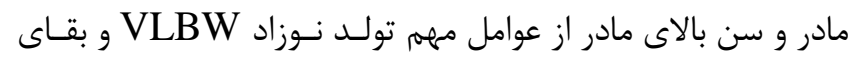

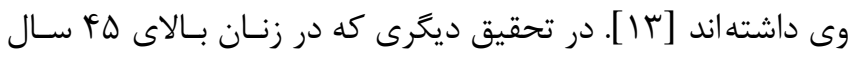

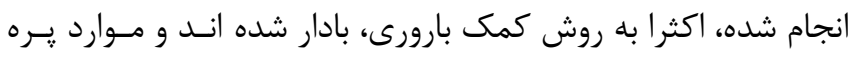

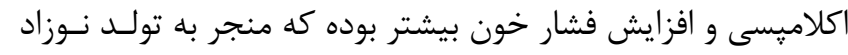
VLBW

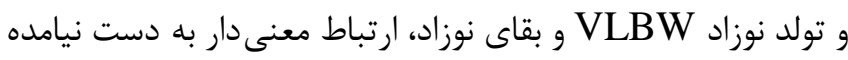

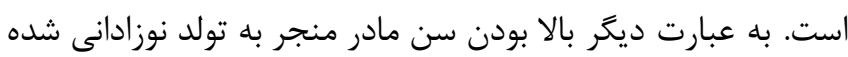

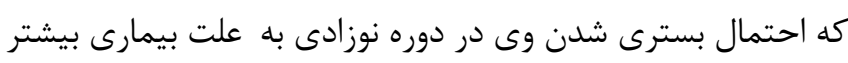

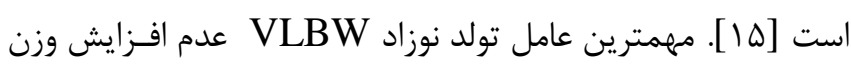

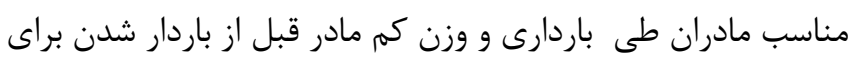

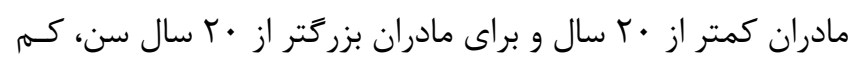

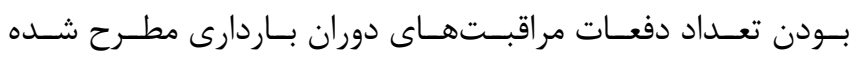


كردهاند [عץ]. همجنين در مطالعه ديخرى در آمريكا ميزان مـرك و و

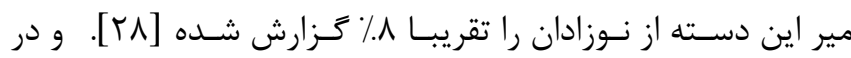

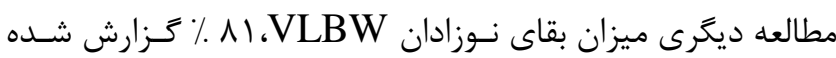

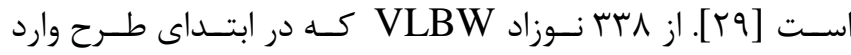

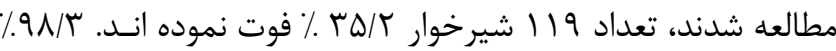

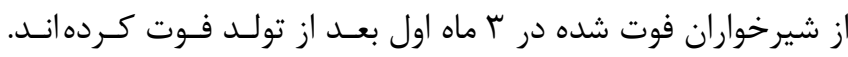

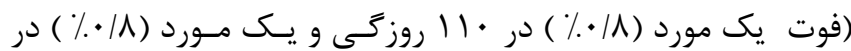

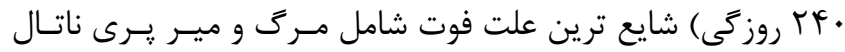

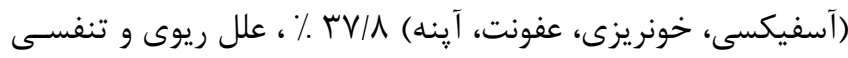

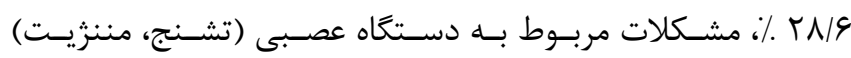

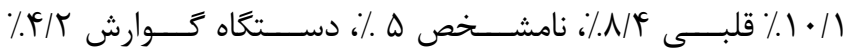

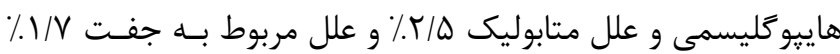

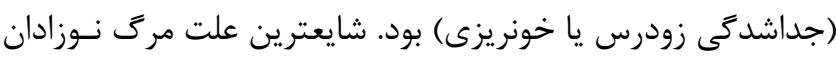

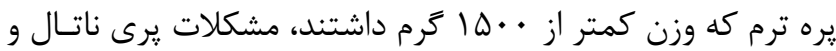

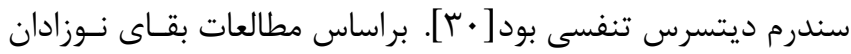

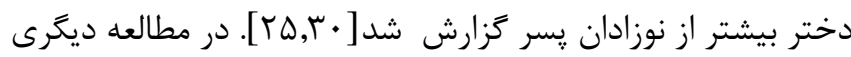

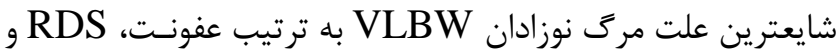

اختلالات مادرزادى كزارش شده [ابّ].

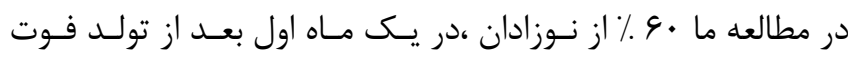

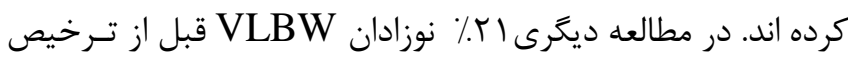

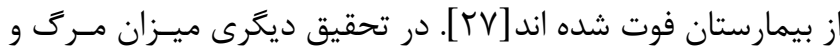

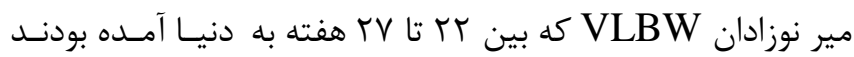

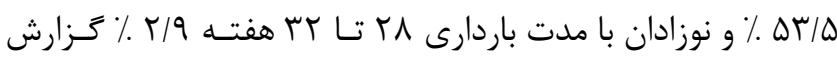

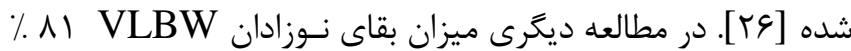

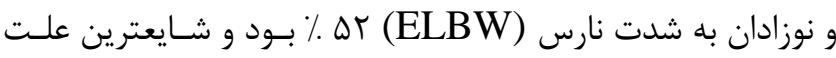

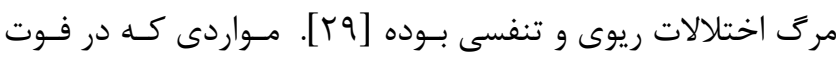

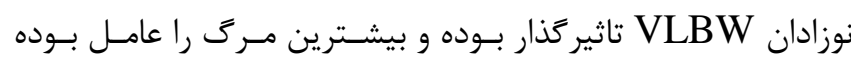

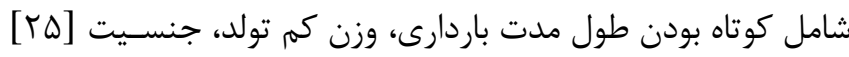

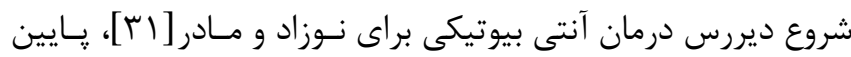

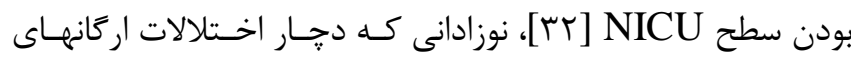

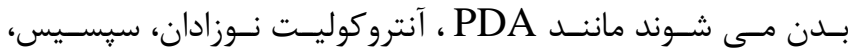

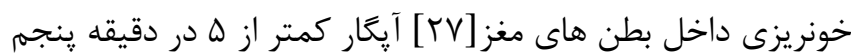

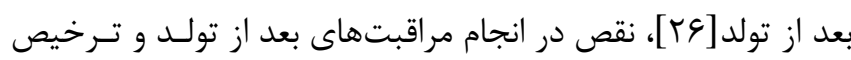

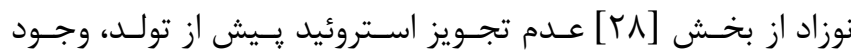

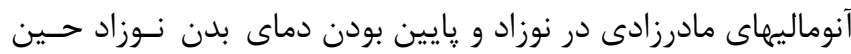

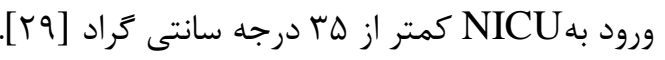

ناتال در موارد تك جفت بودن دوقلويى ؟ برابر دو جفتى است [YT]].

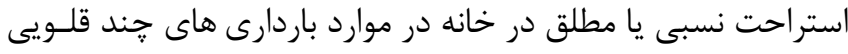

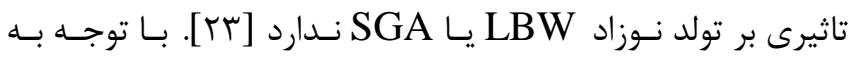

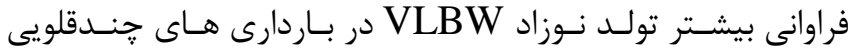
توصيههايى جهت كاهش بروز اين دسـته از بـاردارىهــا مسى شـودي

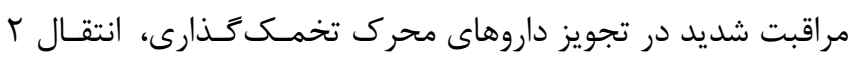

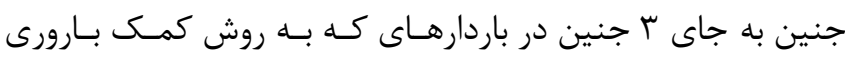

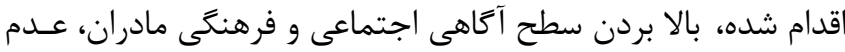

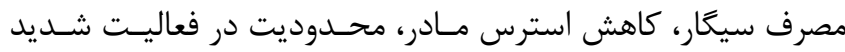

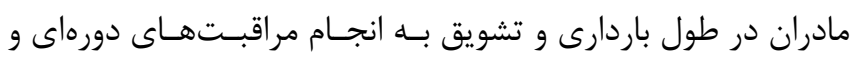

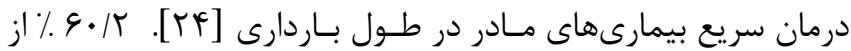
موارد مرك نوزادان

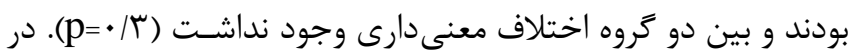

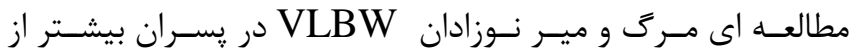

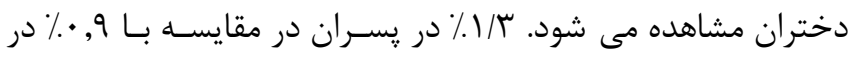

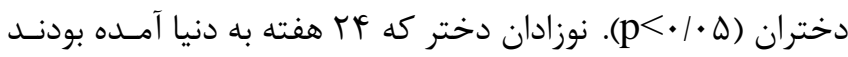

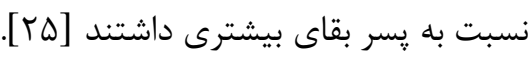
در مطالعه انجام شده بين كم بودن وزن زمان تولد و فـوت نـوزادان بــان

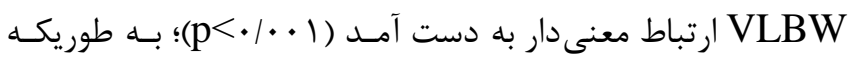

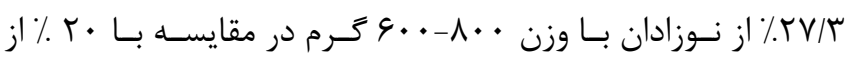

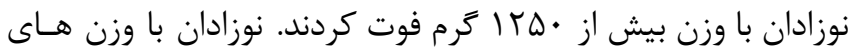

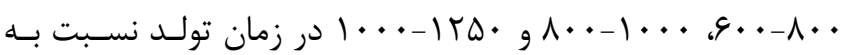

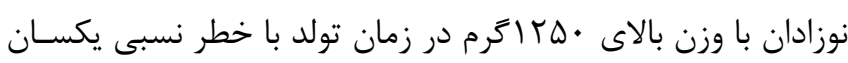

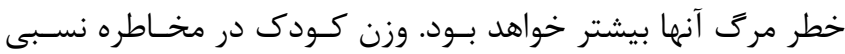
مرى كودى موثر است و اين ميزان براى تمـامى وزن هــاى دسـته

بندى شده به يك ميزان خواهد بود.

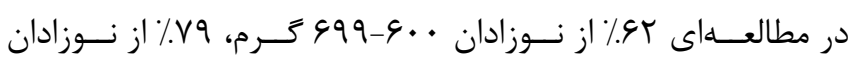

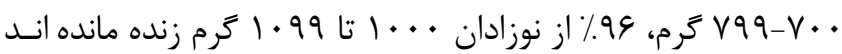

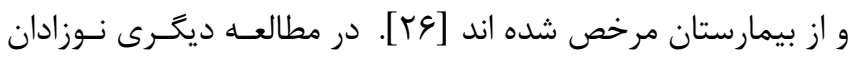

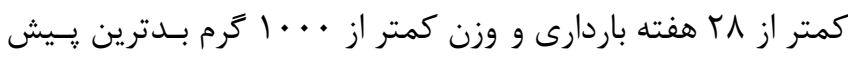

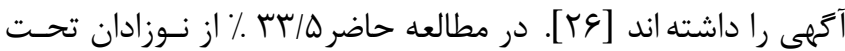

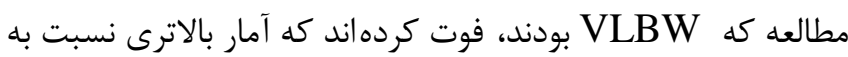

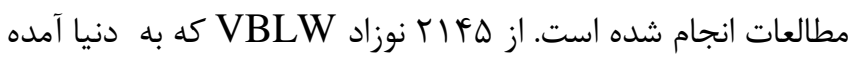

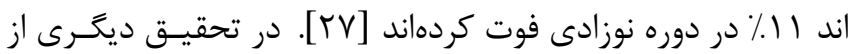

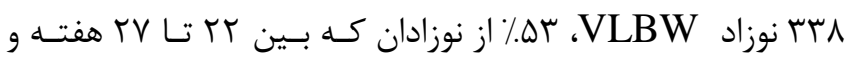

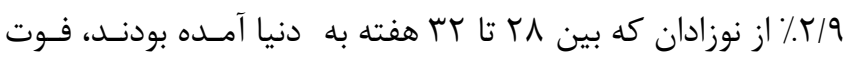




$$
\begin{aligned}
& \text { سهم نويسند } \\
& \text { رامين مظفرى كرمانى: طراحى يزوهش، نغارش مقاله }
\end{aligned}
$$

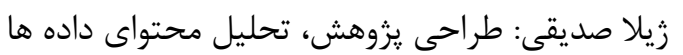

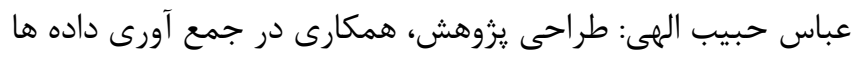

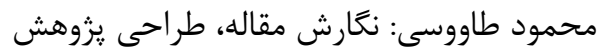

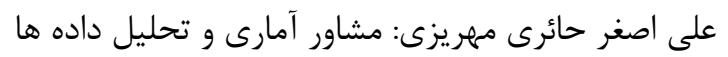

$$
\begin{aligned}
& \text { راحله رستمى: جمع آورى داده، نغارش مقاله } \\
& \text { تشكر و قدردانى }
\end{aligned}
$$

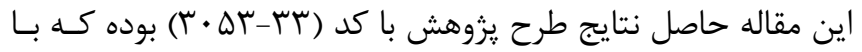

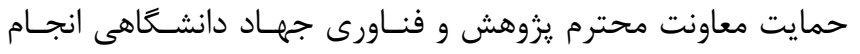

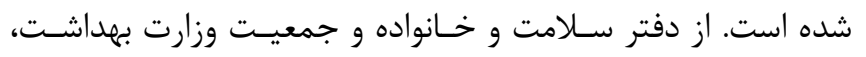

$$
\begin{aligned}
& \text { درمان و آموزش يزشكى كه ما را در اجراى اين مطالعه يارى كردند، } \\
& \text { قدردانى مى شود. }
\end{aligned}
$$

\section{منابع}

1. Kliegman RM, Behrman RE, Jenson HB, Stanton BF. Nelson Textbook of Pediatrics. Saunders Elsevier. $18^{\text {th }}$ Edition: UK, 2008

2. Maureen Kessenich MA. Developmental outcomes of premature, low birth weight, and medically fragile infants. Newborn and Infant Nursing Reviews 2003; 3: 80-87

3. Macquart-Moulin G, Baret C, Julian C, Fancello G, Vincent A, Aymé S. Antenatal surveillance and risks of prematurity and fetal growth retardation. Journal de Gynecologie, Obstetrique et Biologie de la Reproduction (Paris) 1992; 21: 9-18

4. Chaudhari S, Otiv M, Chitale A, Hoge M, Pandit A, Mote A. Biology versus environment in low birth weight children. Indian Pediatrics 2005; 42: 763-7

5. Yaya S, Bishwajit G, Ekholuenetale M, Shah V. Inadequate Utilization of Prenatal Care Services, Socioeconomic Status, and Educational Attainment Are Associated with Low Birth Weight in Zimbabwe. Front Public Health 2017; 5:35

6. Rajizadeh A , Samimi Sh, Momeni M , Investigation of the factors related to low birth weight in the south of Iran. Scientific Journal of Kurdistan University of Medical Science 2018; 23: 46-56 [Persian]

7. Grytten J, Skau I,Sørensen RJ. Educated mothers, healthy infants. The impact of a school

$$
\begin{aligned}
& \text { از نقاط ضعف و محدوديت مطالعه، كاهش تعداد افراد تحت مطالعـهـ }
\end{aligned}
$$

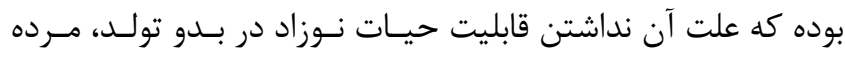

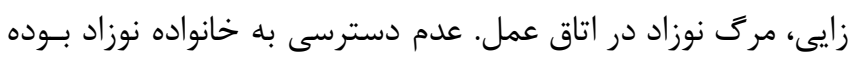

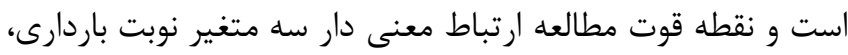

$$
\begin{aligned}
& \text { مدت باردارى و بيمه خانواده با مرك كودكان بوده است. }
\end{aligned}
$$

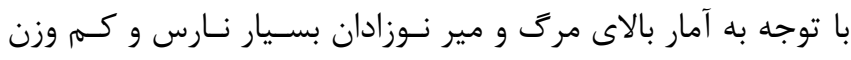

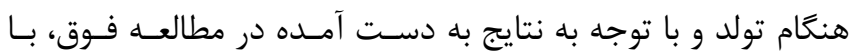

$$
\begin{aligned}
& \text { تشويق مادران به ادامه تحصيل و بالارفتن سـطح تحصـيلات مـادر، }
\end{aligned}
$$

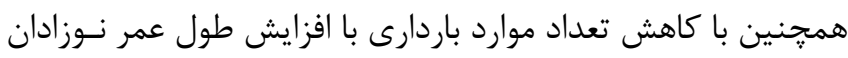

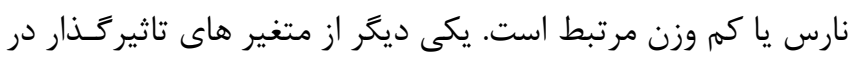

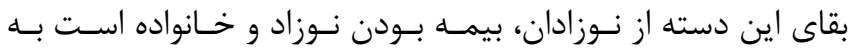

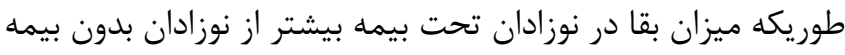

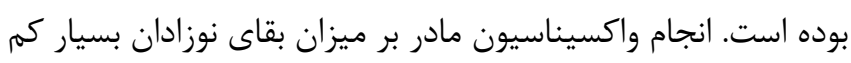

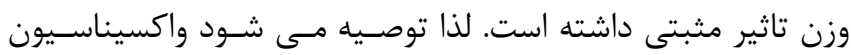

$$
\begin{aligned}
& \text { زنان طبق برنامه كشورى انجام شود. }
\end{aligned}
$$

reform on the birth weight of Norwegian infants 19672005. Social Science \& Medicine $2014 ; 105: 84-92$

8. Boyce LK, Cook GA, Simonsmeier V, Hendershot SM. Academic outcomes of very low birth weight infants: the influence of mother-child relationships. Infant Mental Health Journal 2015; 36:156-66

9. Rogowski J. Cost-effectiveness of care for very low birth weight infants. Pediatrics 1998 ; 102: $35-43$

10. Brokamp C, Beck AF, Goyal NK, Ryan P, Greenberg JM, Hall ES. Material community deprivation and hospital utilization during the first year of life: an urban population-based cohort study. Annual Epidemiology 2019; 30: 37-43

11. Stolz JW, McCormick MC. Restricting access to neonatal intensive care: effect on mortality and economic savings. Pediatrics 1998; 101: 344-8

12. Sydsjö G, Lindell Pettersson M, Bladh M, Skoog Svanberg A, Lampic C, Nedstrand E. Evaluation of risk factors' importance on adverse pregnancy and neonatal outcomes in women aged 40 years or older. BMC Pregnancy Childbirth 2019; 13:92

13. Balázs P, Rákóczi I, Grenczer A, Foley KL. Risk factors of preterm birth and low birth weight babies among Roma and non-Roma mothers: a 
population-based study. European Journal of Public Health 2013; 23: 480-5

14. Avnon $\mathrm{T}$, Haham A, Many A. Twin pregnancy in women above the age of 45 years: maternal and neonatal outcomes. Journal Perinatology Medicine. 2017; 45:787-7

15. Kocourková J, Šídlo L, Št’astná A, Fait T. Impact of the mothers age at childbirth on the birth weight of new-born children. Cas Lek Cesk 2019; 158: 118-125

16. Wang CS, Chou P. Risk factors for low birth weight among first-time mothers in southern Taiwan. Journal of the Formosan Medical Association 2001; 100:168-72

17. Aliyu MH, Salihu HM, Keith LG, Ehiri JE, Islam MA, Jolly PE. High parity and fetal morbidity outcomes. Obstetrics \& Gynecology 2005; 105:1045-5 18. Agarwal P, Sriram B, Rajadurai VS. Neonatal outcome of extremely preterm Asian infants $\leqslant 28$ weeks over a decade in the new millennium. Journal of Perinatology 2015; 35: 297

19. Spiegler J, Stichtenoth G, Weichert J, König IR, Schlaud M, V D Wense A, Olbertz D, Gurth H, Schiffmann JH, Bohnhorst B, Gortner L, Herting E, Göpel W. Pregnancy risk factors for very premature delivery: what role do hypertension, obesity and diabetes play? Archive Gynecology Obstetrics 2013; 288: 57-64

20. Badilla $X$, Morice A, Avila-Aguero ML, Saenz E, Cerda I, Reef S, Castillo-Solórzano C. Fetal risk associated with rubella vaccination during pregnancy. The Pediatric Infectious Disease Journal 2007; 26: 830-5

21. Castillo-Solórzano $\mathrm{C}$, Reef SE, Morice A, Vascones N, Chevez AE, Castalia-Soares R, Torres C, Vizzotti C, Ruiz Matus C. Rubella vaccination of unknowingly pregnant women during mass campaigns for rubella and congenital rubella syndrome elimination, the Americas 2001-2008. International Journal of Infectious Diseases 2011; 2: 713-7

22. Krampl E, Klein K. Prematurity in multiple pregnancies. Gynakol Geburtshilfliche Rundsch 2007; 47: 64-9
23. da Silva Lopes $\mathrm{K}$, Takemoto $\mathrm{Y}$, Ota E, Tanigaki S, Mori R. Bed rest with and without hospitalisation in multiple pregnancy for improving perinatal outcomes. Cochrane Database of Systematic Reviews 2017; 6:3

24. Kurdi AM, Mesleh RA, Al-Hakeem MM, Khashoggi TY, Khalifa HM. Multiple pregnancy and preterm labor. Saudi Medical Journal. 2004; 25: 632-7 25. Jones HP, Karuri S, Cronin CM, Ohlsson A, Peliowski A, Synnes A, Lee SK. Actuarial survival of a large Canadian cohort of preterm infants. BMC Pediatrics 2005; 9: 5:40

26. Jakuskiene R, Vollmer B, Saferis V, Daugeliene D. Neonatal outcomes of very preterm infants admitted to a tertiary center in Lithuania between the years 2003 and 2005. The European Journal of Pediatrics 2011; 170:1293-303

27. Kusuda S, Fujimura M, Sakuma I, Aotani H, Kabe K, Itani Y, Ichiba H, Matsunami K, Nishida H. Morbidity and mortality of infants with very low birth weight in Japan: center variation. Pediatrics 2006; 118: $1130-8$

28. Escobar GJ, Clark RH, Greene JD. Short-term outcomes of infants born at 35 and 36 weeks' gestation: we need to ask more questions. Seminar Perinatology 2006; 30: 28-3

29. Sritipsukho $\mathrm{S}$, Suarod $\mathrm{T}$, Sritipsukho $\mathrm{P}$. Survival and outcome of very low birth weight infants born in a university hospital with level II NICU. Journal of the Medical Association of Thailand 2007; 90:1323-9

30. $\mathrm{Xu}$ FD, Kong XY, Feng ZC. Mortality rate and cause of death in hospitalized neonates: an analysis of 480 cases. Zhongguo Dang Dai Er Ke Za Zhi 2017; 19:152-1.58

31. Lorayne Barton, Joan E. Hodgman and Zdena Pavlova. Causes of Death in the Extremely Low Birth Weight Infant. Pediatrics February 1999;103:446-451 32. Jensen EA, Lorch SA. Effects of a Birth Hospital's Neonatal Intensive Care Unit Level and Annual Volume of Very Low-Birth-Weight Infant Deliveries on Morbidity and Mortality. JAMA Pediatrics 2015; 169: e151906 